Technical Note

\title{
Overview of posterior knee arthroscopy in the medial meniscal repair: Technical note
}

\author{
Sohrab Keyhani, MD ${ }^{\mathrm{a}}$, Arash Sherafat Vaziri, MD ${ }^{\mathrm{b}}$, Fardis Vosoughi, MD ${ }^{\mathrm{b}, *}$, René Verdonk, MD ${ }^{\mathrm{c}}$, \\ Mohammad Movahedinia, MD ${ }^{\text {a }}$ \\ a Bone Joint and Related Tissues Research Center, Akhtar Orthopedic Hospital, Shahid Beheshti University of Medical Sciences, Tehran, Iran \\ ${ }^{\mathrm{b}}$ Department of Orthopaedic and Trauma Surgery, Shariati Hospital, Tehran University of Medical Sciences, Tehran, Iran \\ ${ }^{\mathrm{c}}$ Department of Orthopedic Surgery, ULB University Hospital Erasmus, Brussels, Belgium
}

\section{A R T I C L E I N F O}

\section{Keywords:}

Repair

Enhancement

biologic

subchondral

sagging

trans-septal

ramp

bucket

reduction

preserve

\begin{abstract}
A B S T R A C T
Preservation of the meniscus in bucket handle medial meniscal tears (BHMMTs) and posterior meniscocapsular (ramp) lesions is challenging. Current efforts are being made in the scientific community to (1) introduce new ways of gaining easier and better field of view over the repair site and (2) increase the chance of meniscal healing through effective augmentation procedures. The current note introduces a way to achieve good exposure of posterior knee compartments and proposes an augmentation technique that involves exposing the local nonarticulating subchondral cancellous bone and making in situ clots.
\end{abstract}

\section{Current techniques}

- All-inside repair with/without further enhancement (due to simultaneous ACL reconstruction) with sequential use of the anterior and the posteromedial portal with a cannula along with 30- and 70-degree lenses

- All-inside repair with/without further enhancement through the anterior portals

- All-inside repair with/without further enhancement with the use of two posteromedial portals

\section{New technique}

- Exposure and repair method: All-inside repair through the posterolateral trans septal view and posteromedial working portal

- Enhancement method: focused abrasion of the meniscal undersurface bone with an awl or a burr

\section{Introduction}

The treatment of medial meniscal posterior horn lesions, including those of the peripheral meniscocapsular attachments (also known as ramp lesions) and bucket handle medial meniscal tears (BHMMTs), presents an ongoing matter of debate among knee surgeons. Meniscal repair, especially in the vascular zone, has shown promising results [1]. Resecting as little as $34 \%$ of the meniscal circumference increases contact pressure of the respected compartment up to 3-fold. As such, it is a growing worldwide trend to save the meniscus even in avascular zones [2-4]. A recent study showed that the rate of meniscal repair has doubled during the last decade [5-7].

Partial healing is a frequent phenomenon in tears of the posterior third of the meniscus (areas A and F of the Cooper classification system) (Fig. 1) [8-11]. Merely suturing the meniscal tear will not always result in complete healing. Biologic augmenting techniques have been introduced to achieve better healing, especially in the less vascular areas of the menisci (circumferential zones 2 and 3 of the Cooper system) [2,8]. Rasping or abrasion of the para-meniscal synovium along with the meniscal surface removes local fibrins and brings cytokines and growth factors to the repair site. It can be done easily during arthroscopic repair

\footnotetext{
* Corresponding author. Shariati Hospital, Tehran University of Medical Sciences, Jalal Street, Tehran, Iran. Tel.: +982184901000; Fax: +982188633039.

E-mail addresses: sohrab.keyhani4@gmail.com (S. Keyhani), arash.sharafatvaziri@gmail.com (A.S. Vaziri), Fardis.vosoughi@gmail.com (F. Vosoughi), rene. verdonk@ugent.be (R. Verdonk), Mmvn93@gmail.com (M. Movahedinia).
} 


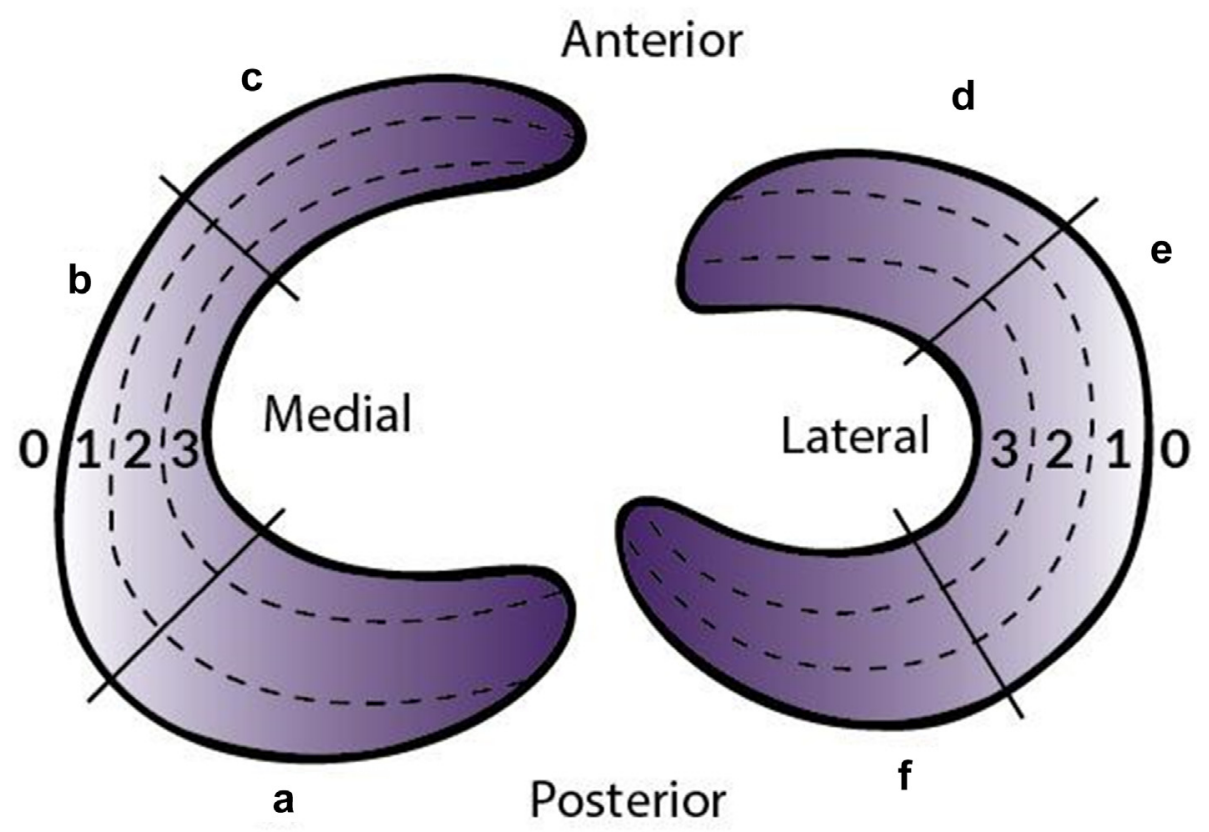

Fig. 1. Cooper's classification of meniscal zones [10].

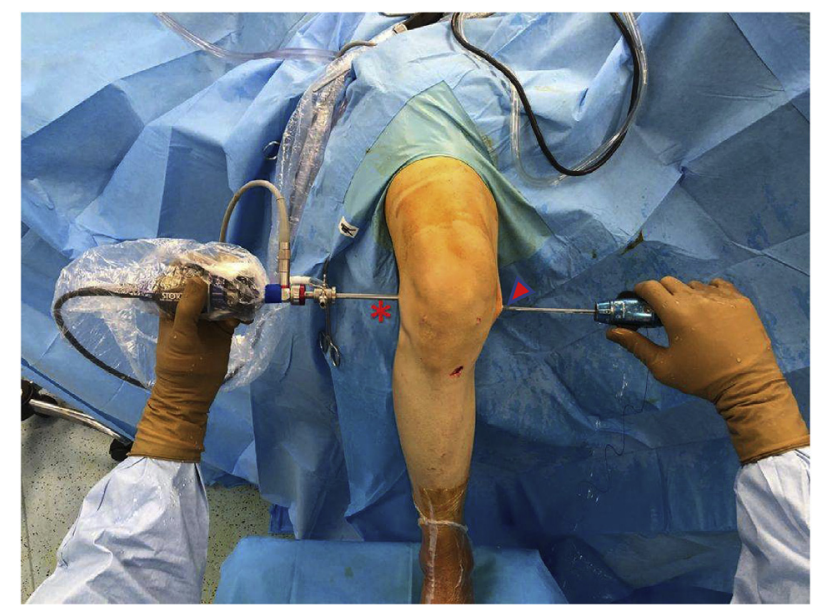

Fig. 2. Right knee:. Position of the limb for posterior trans-septal arthroscopy. Asterisk: posterolateral portal, arrow: posteromedial portal.

and has therefore become one of the most widely used augmentation techniques [2,3]. Trephination, fibrin clot, platelet-rich plasma (PRP), and synovial flap sewn into the tear are other techniques that have been introduced so far [1-3,7,8,12-14].

A recent popular type of augmentation focuses on marrow stimulation to enhance meniscal healing. This method was first proposed when higher rates of successful repair were observed in patients undergoing concomitant anterior cruciate ligament (ACL) reconstruction and meniscal repair $[1,15,16]$. Thereby, bone marrow stimulation was introduced as a method of augmentation for meniscal healing in isolated tears [7].

Most areas in the posterior compartment of the knee cannot be easily visualized with anterior portals $[17,18]$. Some surgeons release the medial collateral ligament (MCL) percutaneously to open the medial compartment and prevent chondral injury [19]. However, adequate rasping of the meniscosynovial junction and meniscal surface at the most posterior areas of a teared meniscus is still challenging [20-22].

This technical note presents posterior arthroscopy (using posterior portals with trans-septal view) as a decent method of access to zone A of the medial meniscus. It also introduces a new method of biologic augmentation and bone marrow stimulation that involves exposing nonarticular subchondral bone of the posterior tibial rim during the repair of ramp lesions or BMMMTs.

\section{Technique}

The patient is placed supine on the operating table with a tourniquet placed high on the thigh. We remove the foot of the bed so that the leg is hanging from the end of the bed (Fig. 2). Good access to posterior portals is achieved in this manner.

Arthroscopic knee examination is performed through the standard anterior portals. Then, through the trans-notch view, medial to the cruciate ligaments, the posteromedial compartment is visualized via the trans-notch path. If the medial meniscal tear is seen or suspected, and for all BHMMTs after reduction, the posterolateral trans-septal view [7] is planned.

Posterior trans-septal arthroscopy requires creating both the posterolateral and posteromedial portals. The incision for posterior portals is made with the guidance of transillumination. Attention is paid to avoid the popliteal bundle and the common peroneal nerve. The posterolateral portal location is found just by the lateral femoral condyle with an 18-gauge needle, followed by incision with a number11 blade. The posterolateral portal is created in the soft spot area under direct vision from the anteromedial portal. Afterwards, the septum between the posteromedial and posterolateral knee compartments is penetrated with a blunt instrument, such as an arthroscopic sheath and trocar. The point of penetration in the septum is critical: it should be just posterior to posterior cruciate ligament (PCL) at the midpoint in the vertical direction. If it is too high, it could cause bleeding from the arterial branches of the middle geniculate artery, and, if too low, it could restrict the surgeon's vision through the trans-septal portal along with risking injury to the popliteal vessels (Figs. 3 and 4). Similarly, anterior placement of the portal results in difficult access and might cause lateral femoral condyle chondral injury, while posterior placement endangers the neurovascular bundle behind the knee capsule. After passing through the septum, the lens is transferred to the posterolateral trans-septal portal, providing an excellent view of the posterior aspect of medial meniscus. The posteromedial portal can now be created by introducing an 18-gauge needle in an outside-in fashion in 


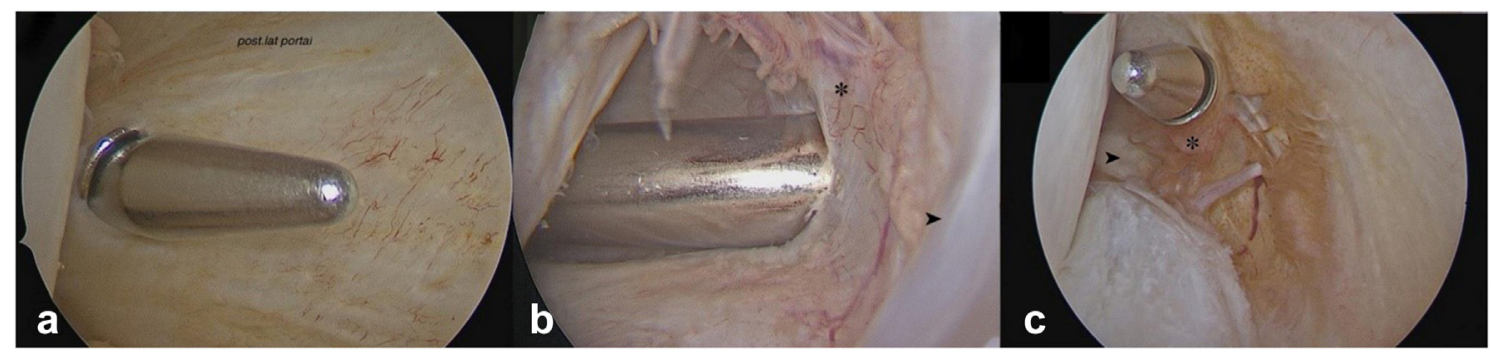

Fig. 3. Right knee: posterolateral, trans-septal, and posteromedial portal creation: (A) The location of the posterolateral portal, by trans notch view. (B) Arthroscopic sheet from the posterolateral portal passed through the posterior septum by hand pressure without septum shaving. (C) Posteromedial portal view of the septum. (Arrow head: PCL; Asterisk: septum).

a soft spot just by the posteromedial edge of the femoral condyle. There is no need for a $70^{\circ}$ lens with this method. There is full access to the posterior third of the medial meniscus from the root area up to the corner point, which is between the posterior and middle thirds of the medial meniscus at the insertion point of the semimembranous direct head $[7,17,18,21,23,24]$. Although it is occasionally useful, using the

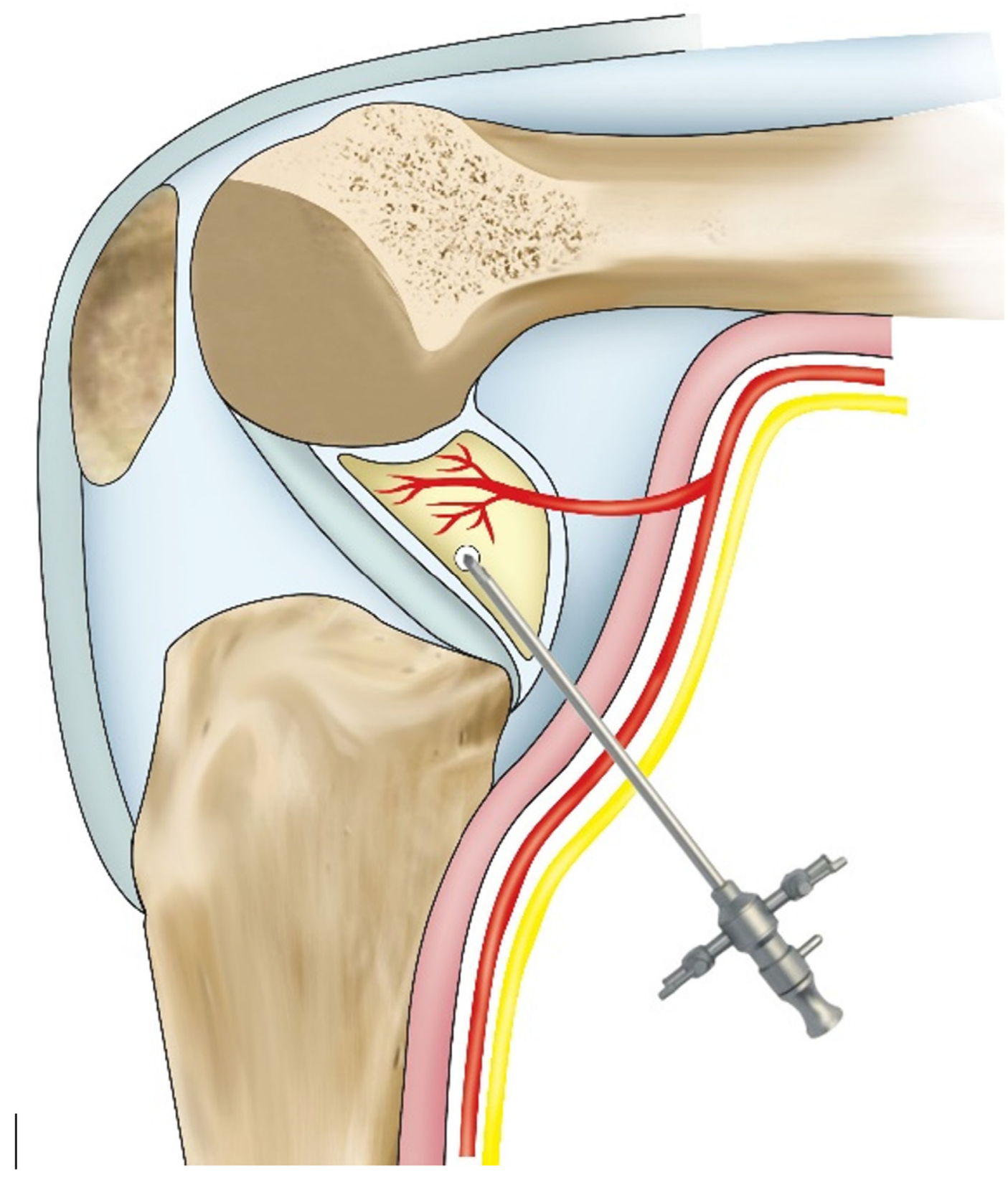

Fig. 4. Schematic drawing illustrating the creation of the trans-septal portal during posterior arthroscopy of the knee. (This figure was created by the authors for the sole purpose of the current study.) 


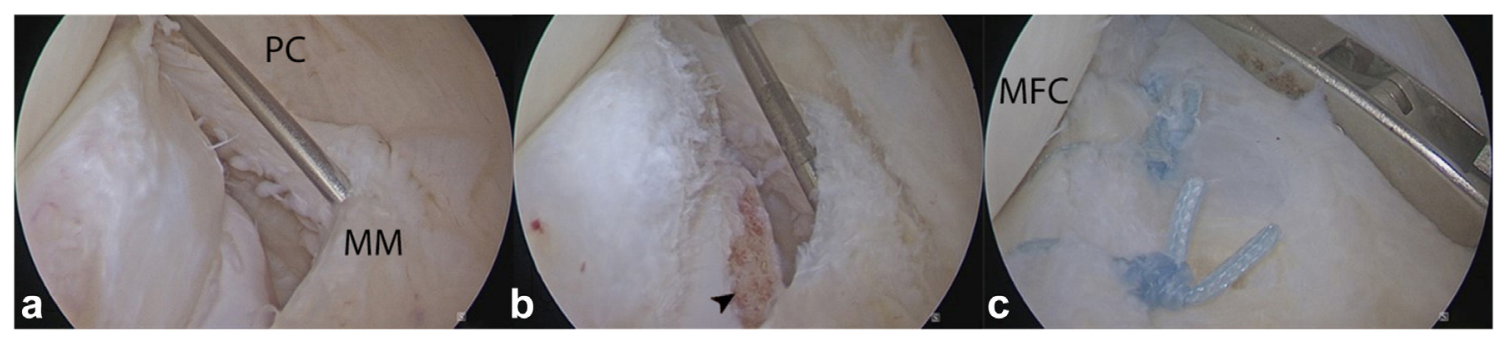

Fig. 5. Left knee: Posterolateral trans-septal view of the ramp lesion, before (A) and after abrasion chondroplasty-arrow head (B) and complete repair (C). (PC: posterior capsule, MM: medial meniscus, MFC: medial femoral condyle).

$70^{\circ}$ lens through the notch would not give a great view of the posterior corner point, and trying to use it in this area might lead to the risk of device breakage [25].

Our technique involves a three-step augmentation procedure before proceeding to meniscal repair:

1. Thorough débridement: Complete removal of the fibrotic tissue at the posterior meniscosynovial junction (zone A of the medial meniscus). This is done with use of a non-aggressive arthroscopic shaver without suction to avoid the removal of healthy tissue. Red-red and red-white zones of the medial meniscus are completely approached for removal of the fibrotic tissue to reach healthy tissue. Removal of some avascular free edges (small flap tears) from both sides of the tear is mandatory and easily possible.

2. Rasping: A meniscal rasp with a suitable angle is introduced from the posteromedial portal for abrasion of both the synovial and meniscal sides of the tear under direct vision from posterolateral trans-septal view (Fig. 5).

3. Biologic augmentation: For in-situ clot formation (the most important part of the augmentation procedure), an arthroscopic 4.5- or 5-mm burr is introduced from the posteromedial portal to completely abrade the bony rim of the posteromedial tibial plateau until bleeding subchondral cancellous bone is exposed. The exact target is the posteromedial border of the tibial plateau, which is not weightbearing and does not contain articular cartilage. In some cases, especially in smaller knees, we use a microfracture awl to create active bleeding holes. These attempts result in the development of an in-situ clot at the inferior surface of the repair site after releasing the tourniquet (Fig. 5B).

After fully preparing the meniscus borders and finishing the augmentation procedure, the repair is started from the posteromedial as a working portal while viewing from the posterolateral trans-septal portal. A suture lasso is used to create vertical mattress sutures (Fig. 5C). The repair also can be performed from a working posteromedial portal under the view of anterolateral notch view or working anteromedial portal under view from the anterolateral portal with an all-inside or inside-out method depending upon the configuration of the meniscal tear or surgeon's preference. In certain cases, especially when there is inferior sagging of the peripheral posterior fragment (Figs. 6C and 8), repair from the posteromedial portal helps us to correct the meniscal deformity by elevating the peripheral fragment and reaching anatomical reduction, which will, in turn, increase the chances of meniscal healing [19].

\section{Discussion}

More than half (51.9\%-63\%) of knees with an ACL tear are found to have a concomitant meniscal tear [26,27]. Today, bucket handle medial meniscus tears (BHMMTs) and ramp lesions are more commonly diagnosed than ever before [28]. Numerous biomechanical studies have shown meniscal repair to have superior short- and long-term outcomes compared with partial or subtotal meniscectomy [29]. There is no consensus on the success rate of meniscus repair among knee surgeons, but most agree that some type of augmentation is necessary for accelerating the healing process at the repair site [1-3,6-10,12-14,20,29-36].

Various techniques have been introduced to enhance the healing process. Trephination was thought to be effective as it provided vascular pathways between the red-red zone and the more central zones. But this happens at the expense of cutting circumferential collagen fibers, which makes surgeons hesitant to implement it [37-39]. Blood and bone marrow have been shown to contain growth factors, which might account for improved meniscal healing in the cases involving concomitant ACL reconstruction and tunnel preparations. Nevertheless, it is not clear how much of these released growth factors reach the undersurface of the meniscus, especially in the posterior capsular attachments of the medial meniscus. Interestingly, this is a common site of incomplete healing [40]. Therefore, we propose local augmentation of meniscal healing by providing a source of direct bleeding and possible on-site clot formation by exposing the local subchondral bone under the tear site.

Several studies have shown that meniscus rasping both at the meniscal and para-meniscal synovial side can be advantageous by: [2,3] (1) removal of any fibrous tissue at the tear site that prohibits healing of the torn edges; and (2) providing several cytokines and growth factors such as interleukin-1, transforming growth factor (TGF), and platelet-derived growth factor (PDGF), on the rasped area within the first

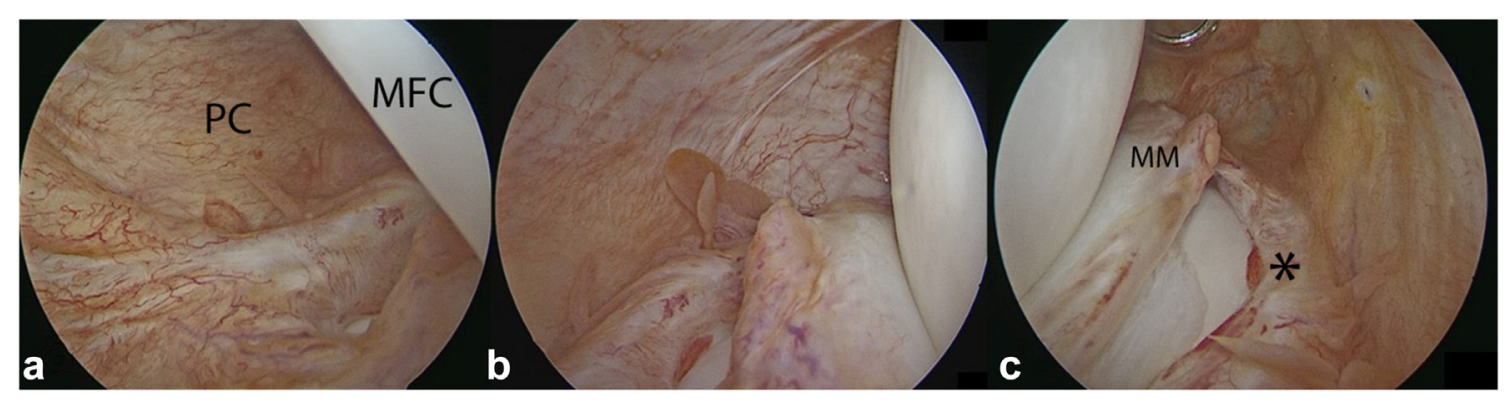

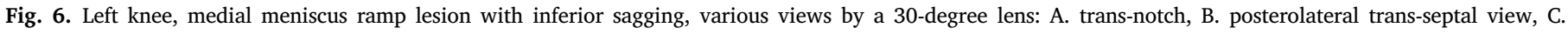
posteromedial view. (PC: posterior capsule, MM: medial meniscus, MFC: medial femoral condyle) [6]. 


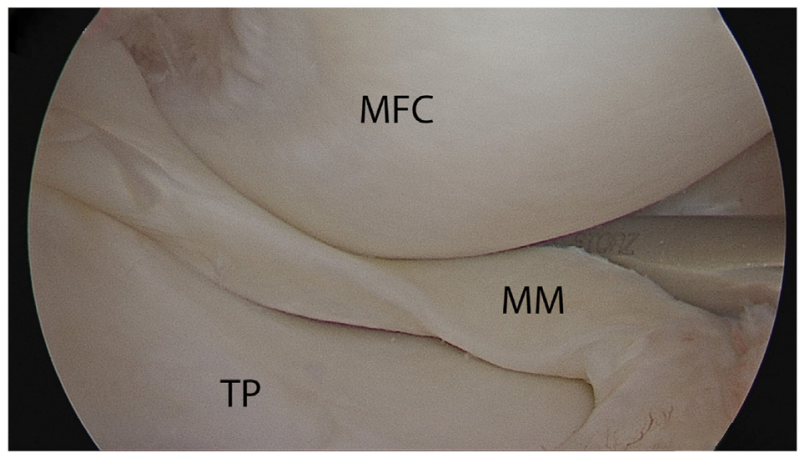

Fig. 7. Right knee, anterolateral portal view: Arthroscopic abrasion of torn meniscal borders with shaver before reduction (TP: tibial plateau, MM: medial meniscus, MFC: medial femoral condyle).

60 days postoperatively. Such rasping not a demanding technique and can be done easily during knee arthroscopy with mechanical or motorized instruments. Hence, we prefer to perform shaver abrasion in all repairs. Accessing the most posterior part of the medial meniscus is best achieved by means of posterior arthroscopy [7,19].

BHMMTs and ramp repairs usually accompany an ACL reconstruction, which releases bone marrow into the joint. Incomplete healing after medial meniscal repairs, especially in the posterior horn, has been reported, leaving the undersurface of the meniscus last to heal (if at all) [40]. This occurs probably because of insufficient local concentration of growth factors at the undersurface of the medial meniscus [40]. We believe that rasping through posterior arthroscopy could be an effective tool for releasing the local stimulating factors that augment the repair process.
When treating BHMMTs, we prefer to abrade the meniscal borders before reduction with use of a motorized shaver or rasp, as the edges are usually everted (Fig. 7). When using anterior portals alone for rasping the synovial side of the posterior meniscus, vigorous MCL release might be necessary to open up the medial compartment and to prevent iatrogenic cartilage injury. This can easily be avoided by using posterior arthroscopy [19]. We never reduce the BHMMT before completely rasping the meniscal borders, as the reduced meniscus itself will block our access to the synovial side. Furthermore, care must be taken not to over-release the MCL as it might cause postoperative pain or knee laxity [19]. Therefore, we prefer to abrade the posterior synovium from the posteromedial portal while observing from a trans-notch or trans-septal view [19]. In this manner, we will have a great view of the posteromedial compartment without any barriers and we can abrade the synovial and inner borders of the peripheral fragment freely, irrespective of whether the meniscus is reduced or not.

There are two types of posterior medial meniscal tears: with or without inferior sagging of the peripheral fragment. In the first group, a repair can be performed with either anterior or posterior arthroscopy. In the latter group, in which the peripheral segment of the meniscus sags inferiorly, repair is not easily possible through the anterior approach (Fig. 8). Posterior arthroscopy is the best solution to bring this peripheral lower fragment to the level of the central upper fragment and provide maximal contact surface. In either group of tears, due to the ease of access, less risk of chondral injury, and the possibility of more stable repairs with vertical mattress sutures, the authors' preference is the posterior arthroscopy through the trans septal approach [19].

In conclusion, our preferred technique of repair in zone $\mathrm{A}$ of the medial meniscus is the all-inside repair from the posteromedial portal with a suture lasso for applying vertical mattress sutures with higher biomechanical strength. This technique is especially helpful in cases in

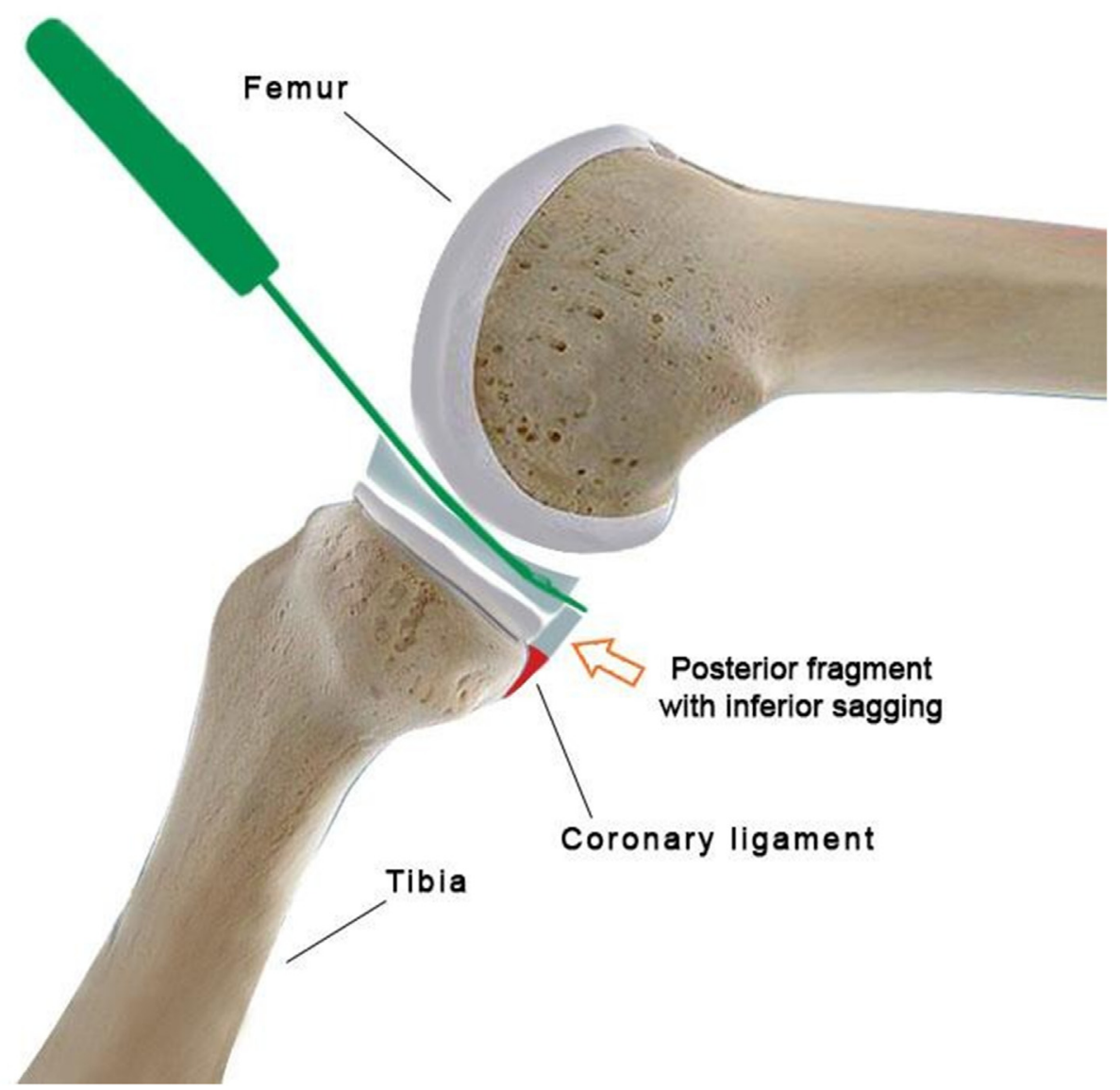

Fig. 8. Schematic drawing showing the inaccessibility of an inferiorly sagging posterior fragment through the anterior arthroscopy. 
which inferior sagging of the posterior fragment is encountered. We propose performing posterior abrasion of the synovium with use of a shaver or rasp and chondroplasty of the posteromedial tibial plateau rim with use of a burr from the posteromedial portal in all cases, irrespective of the repair technique. We believe that even during inside-out or allinside repair techniques that are performed through anterior portals, abrasion must be done directly from a posterior portal to achieve the best results.

\section{Declaration of competing interest}

The authors declare that they have no known competing financial interests or personal relationships that could have appeared to influence the work reported in this article.

\section{Acknowledgment}

We would like to thank Saeed Keyhani for preparation of the schematic drawings.

\section{Appendix A. Supplementary data}

Supplementary data to this article can be found online at https ://doi.org/10.1016/j.jisako.2022.02.002.

\section{References}

[1] Acosta J, Ravaei S, Brown SM, Mulcahey MK. Examining techniques for treatment of medial meniscal ramp lesions during anterior cruciate ligament reconstruction: A systematic review. Arthrosc J Arthrosc Relat Surg 2020;36(11):2921-33. https:// doi.org/10.1016/j.arthro.2020.05.041.

[2] Okuda K, Ochi M, Shu N, Uchio Y. Meniscal rasping for repair of meniscal tear in the avascular zone. Arthroscopy 1999;15(3):281-6. https://doi.org/10.1016 s0749-8063(99)70035-6.

[3] Ochi M, Uchio Y, Okuda K, Yamaguchi H, Sakai Y. Expression of cytokines after meniscal rasping to promote meniscal healing. Arthroscopy 2001;17(7):724-31. https://doi.org/10.1053/jars.2001.23583.

[4] Baratz ME, Fu FH, Mengato R. Meniscal tears: The effect of meniscectomy and of repair on intraarticular contact areas and stress in the human knee: A preliminary report. Am J Sports Med 1986;14(4):270-5. https://doi.org/10.1177/ 036354658601400405.

[5] Tashiro Y, Mori T, Kawano T, et al. Meniscal ramp lesions should be considered in anterior cruciate ligament-injured knees, especially with larger instability or longer delay before surgery. Knee Surg Sports Traumatol Arthrosc 2020;28(11):3569-75. https://doi.org/10.1007/s00167-020-06161-8.

[6] Thaunat M, Fayard JM, Guimaraes TM, Jan N, Murphy CG, Sonnery-Cottet B. Classification and surgical repair of ramp lesions of the medial meniscus. Arthrosc Tech 2016;5(4):e871-5. https://doi.org/10.1016/j.eats.2016.04.009.

[7] Keyhani S, Ahn JH, Verdonk R, Abbasian M. Arthroscopic all-inside ramp lesion repair using the posterolateral transseptal portal view. Knee Surg Sports Traumatol Arthrosc 2017;25(2):454-8. https://doi.org/10.1007/s00167-016-4410-9.

[8] Noyes FR, Barber-Westin SD. Arthroscopic repair of meniscal tears extending into the avascular zone in patients younger than twenty years of age. Am J Sports Med 2002;30(4):589-600. https://doi.org/10.1177/03635465020300042001.

[9] van Trommel MF, Simonian PT, Potter HG, Wickiewicz TL. Different regional healing rates with the outside-in technique for meniscal repair. Am J Sports Med 1998;26(3):446-52. https://doi.org/10.1177/2F03635465980260031901.

[10] Cooper DE, Arnoczky SP, Warren RF. Meniscal repair. Clin Sports Med 1991;10(3): 529-48. https://doi.org/10.1016/S0278-5919(20)30608-6.

[11] Rao AJ, Erickson BJ, Cvetanovich GL, et al. The meniscus-deficient knee: Biomechanics, evaluation, and treatment options. Orthop J Sports Med 2015;3(10). https://doi.org/10.1177/2F2325967115611386. 2325967115611386.

[12] Malinowski K, Góralczyk A, Hermanowicz K, Laprade RF. Tips and pearls for allinside medial meniscus repair. Arthrosc Tech 2019;8(2):e131-9. https://doi.org/10 1016/j.eats.2018.10.009.

[13] Morgan CD. The "all-inside" meniscus repair. Arthroscopy 1991;7(1):120-5. https://doi.org/10.1016/0749-8063(91)90093-d.

[14] Thaunat M, Fournier G, O'Loughlin P, et al. Clinical outcome and failure analysis of medial meniscus bucket-handle tear repair: A series of 96 patients with a minimum 2 year follow-up. Arch Orthop Trauma Surg 2020;140(11):1649-54. https:// doi.org/10.1007/s00402-020-03346-1.

[15] Bollen S. Posteromedial meniscocapsular injury associated with rupture of the anterior cruciate ligament: A previously unrecognised association. J Bone Joint Surg Br 2010;92(2):222-3. https://doi.org/10.1302/0301-620X.92B2.22974.
[16] Liu X, Zhang H, Feng $\mathrm{H}$, et al. Is it necessary to repair stable ramp lesions of the medial meniscus during anterior cruciate ligament reconstruction? A prospective randomized controlled trial. Am J Sports Med 2017;45(5):1004-11. https:// doi.org/10.1177/0363546516682493.

[17] Ahn JH, Ha CW. Posterior trans-septal portal for arthroscopic surgery of the knee joint. Arthroscopy 2000;16(7):774-9. https://doi.org/10.1053/jars.2000.7681.

[18] Kim SJ, Song HT, Moon HK, Chun YM, Chang WH. The safe establishment of a transseptal portal in the posterior knee. Knee Surg Sports Traumatol Arthrosc 2011; 19(8):1320-5. https://doi.org/10.1007/s00167-011-1429-9.

[19] Keyhani S, Soleymanha M, Verdonk R, et al. Posterior knee arthroscopy facilitates the safe and effective all-inside repair of locked bucket-handle medial meniscal tear using a suture hook technique. Knee Surg Sports Traumatol Arthrosc 2021. https:// doi.org/10.1007/s00167-021-06576-x.

[20] Kongmalai P, Chernchujit B. Posterior horn of medial meniscal peripheral capsular lesion: The arthroscopic repair technique working in the posterior compartment. Arthrosc Tech 2016;5(4):e763-7. https://doi.org/10.1016/j.eats.2016.03.001.

[21] Ohishi T, Takahashi M, Suzuki D, Matsuyama Y. Arthroscopic approach to the posterior compartment of the knee using a posterior transseptal portal. World $\mathrm{J}$ Orthoped 2015;6(7):505-12. https://doi.org/10.5312/wjo.v6.i7.505.

[22] Sonnery-Cottet B, Conteduca J, Thaunat M, et al. Hidden lesions of the posterior horn of the medial meniscus: A systematic arthroscopic exploration of the concealed portion of the knee. Am J Sports Med 2014;42(4):921-6. https://doi.org/10.1177/ 0363546514522394.

[23] Ahn JH, Wang JH, Yoo JC, Noh HK, Park JH. A pull out suture for transection of the posterior horn of the medial meniscus: Using a posterior trans-septal portal. Knee Surg Sports Traumatol Arthrosc 2007;15(12):1510-3. https://doi.org/10.1007/ s00167-007-0310-3.

[24] Louisia S, Charrois O, Beaufils P. Posterior "back and forth" approach in arthroscopic surgery on the posterior knee compartments. Arthroscopy 2003;19(3): 321-5. https://doi.org/10.1053/jars.2003.50082.

[25] Lubowitz JH, MacKay G, Gilmer B. Knee medial collateral ligament and posteromedial corner anatomic repair with internal bracing. Arthrosc Tech 2014; 3(4):e505-8. https://doi.org/10.1016/j.eats.2014.05.008.

[26] Cain Jr EL, Fleisig GS, Ponce BA, et al. Variables associated with chondral and meniscal injuries in anterior cruciate ligament surgery. J Knee Surg 2017;30(7): 659-67. https://doi.org/10.1055/s-0036-1593875.

[27] Michalitsis S, Vlychou M, Malizos KN, Thriskos P, Hantes ME. Meniscal and articular cartilage lesions in the anterior cruciate ligament-deficient knee: Correlation between time from injury and knee scores. Knee Surg Sports Traumatol Arthrosc 2015;23(1):232-9. https://doi.org/10.1007/s00167-013-2497-9.

[28] Keyhani S, Esmailiejah AA, Mirhoseini MS, et al. The prevalence, zone, and type of the meniscus tear in patients with anterior cruciate ligament (ACL) injury; does delayed ACL reconstruction affects the meniscal injury? Arch Bone Jt Surg 2020; 8(3):432-8. https://doi.org/10.22038/abjs.2019.39084.2076.

[29] Samuelsen BT, Johnson NR, Hevesi M, et al. Comparative outcomes of all-inside versus inside-out repair of bucket-handle meniscal tears: A propensity-matched analysis. Orthop J Sports Med 2018;6(6):2325967118779045. https://doi.org/ $10.1177 / 2325967118779045$

[30] Alabi IA, Bancha C, Artha A, Kanokvaleewong C, Pena RJ, et al. Arthroscopic procedure for revision of failed isolated bucket-handle meniscal tear repair. J Orthop 2020;21:350-69. https://doi.org/10.1016/j.jor.2020.07.001.

[31] Buyukdogan K, Laidlaw MS, Miller MD. Meniscal ramp lesion repair by a transseptal portal technique. Arthrosc Tech 2017;6(4):e1379-86. https://doi.org/ 10.1016/j.eats.2017.05.029.

[32] Lee WQ, Gan JZW, Lie DTT. Save the meniscus-Clinical outcomes of meniscectomy versus meniscal repair. J Orthop Surg (Hong Kong) 2019;27(2): 2309499019849813. https://doi.org/10.1177/2309499019849813.

[33] Li WP, Chen Z, Song B, Yang R, Tan W, et al. The FasT-Fix repair technique for ramp lesion of the medial meniscus. Knee Surg Relat Res 2015;27(1):56-60. https:// doi.org/10.5792/ksrr.2015.27.1.56.

[34] Okazaki Y, Furumatsu T, Okazaki Y, et al. Medial meniscus posterior root repair decreases posteromedial extrusion of the medial meniscus during knee flexion. Knee 2020;27(1):132-9. https://doi.org/10.1016/j.knee.2019.09.005.

[35] Rothermel SD, Smuin D, Dhawan A. Are outcomes after meniscal repair age dependent? A systematic review. Arthrosc J Arthrosc Relat Surg 2018;34(3): 979-87. https://doi.org/10.1016/j.arthro.2017.08.287.

[36] Wang KH, Hwang DH, Cho JH, Changale SD, Woo SJ, Nha KW. Arthroscopic direct repair for a complete radial tear of the posterior root of the medial meniscus. Clin Orthop Surg 2011;3(4):332-5. https://doi.org/10.4055/cios.2011.3.4.332.

[37] Fox JM, Rintz KG, Ferkel RD. Trephination of incomplete meniscal tears. Arthroscopy 1993;9(4):451-5. https://doi.org/10.1016/s0749-8063(05)80321-4.

[38] Kaminski R, Maksymowicz-Wleklik M, Kulinski K, Kozar-Kaminska K, DabrowskaThing A, Pomianowski S. Short-term outcomes of percutaneous trephination with a platelet rich plasma intrameniscal injection for the repair of degenerative meniscal lesions. A prospective, randomized, double-blind, parallel-group, placebocontrolled study. Int J Mol Sci 2019;20(4):856. https://doi.org/10.3390/ ijms20040856.

[39] Shelbourne KD, Gray T. Meniscus tears that can be left in situ, with or without trephination or synovial abrasion to stimulate healing. Sports Med Arthrosc Rev 2012;20(2):62-7. https://doi.org/10.1097/JSA.0b013e318243265b.

[40] Abolaji Alabi I, Chernchujit B, Kanokvaleewong C, Artha A, Pena RJ. Arthroscopic procedure for chronic isolated bucket-handle meniscal tears. Arthrosc Tech 2021; 10(2):e375-83. https://doi.org/10.1016/j.eats.2020.10.011. 